\title{
Composition Modulation of Pt-based Nanowire Electrocatalysts Enhances Methanol Oxidation Performance
}

Qing-Xia Chen,,${ }^{\mathrm{a} \dagger}$ Cheng-Xin Yu, ${ }^{\mathrm{b}, \dagger}$ Hui-Hui Li, ${ }^{\mathrm{a}}$ Zhen He, ${ }^{\mathrm{a}}$ and Jian-Wei Liu*,a

${ }^{a}$ Hefei National Laboratory for Physical Sciences at the Microscale, Collaborative Innovation Centre of Suzhou Nano Science and Technology, Department of Chemistry, University of Science and Technology of China, Hefei 230026, China. ${ }^{b}$ School of Physics and Materials Science, Anhui University, Hefei 230601, China.

\section{Contents}

\section{Supplementary Figures}

Figure S1. TEM images of three 1D Pt-based catalysts on large area.

Figure S2. EDS spectra of three 1D Pt-based catalysts.

Figure S3. XRD patterns of three 1D Pt-based catalysts.

Figure S4. CV profiles of three 1D Pt-based catalysts.

Figure S5. CV profiles of these catalysts for MOR in Ar-saturated $0.5 \mathrm{M} \mathrm{HClO}_{4}$ solution at a sweep rate of $50 \mathrm{mV} \mathrm{s}^{-1}$.

Figure S6. TEM image of PtRuAgTe NTs after CV activation.

Figure S7. TEM images of PtRuAgTe NTs (a) after MOR measurement and (b) a stability cycle of chronoamperometry. (c) XRD pattern of PtRuAgTe NTs after MOR measurement. Figure S8. XPS of Te 3d orbital in three 1D Pt-based catalysts.

Figure S9. High resolution synchrotron radiation photoelectron spectroscopy.

\section{Supplementary Tables}

Table S1. Electrochemical performance for MOR. 


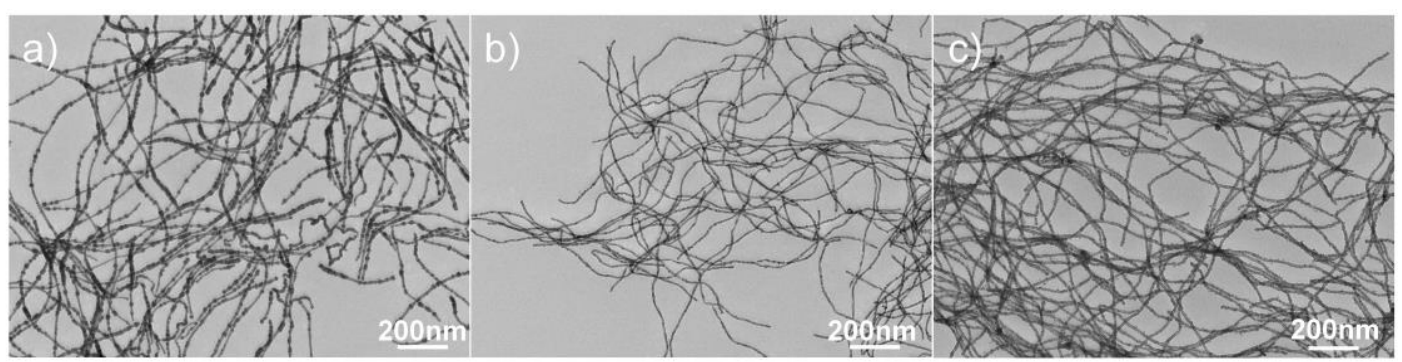

Figure S1. TEM images of three 1D Pt-based catalysts on large area. (a) PtTe NWs, (b) PtAgTe NWs and (c) PtRuAgTe NTs.
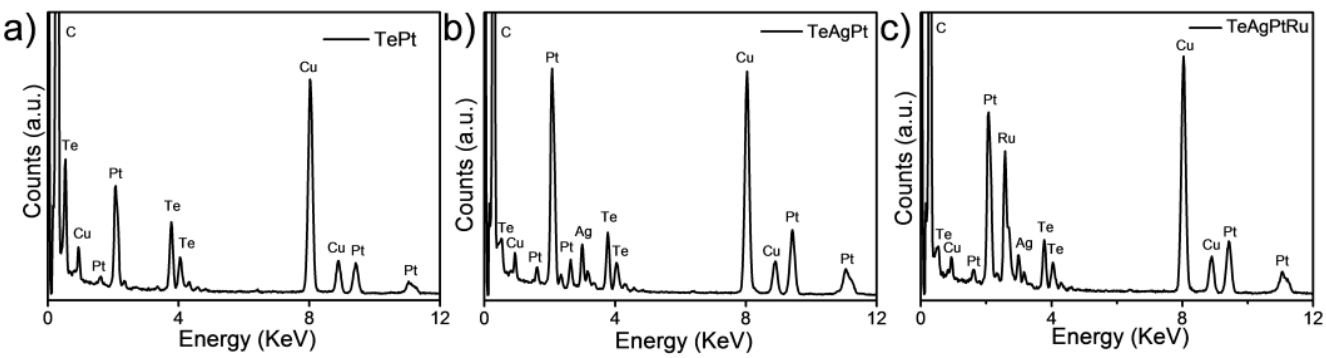

Figure S2. EDS spectra of three 1D Pt-based catalysts. (a) PtTe NWs, (b) PtAgTe NWs and (c) PtRuAgTe NTs.
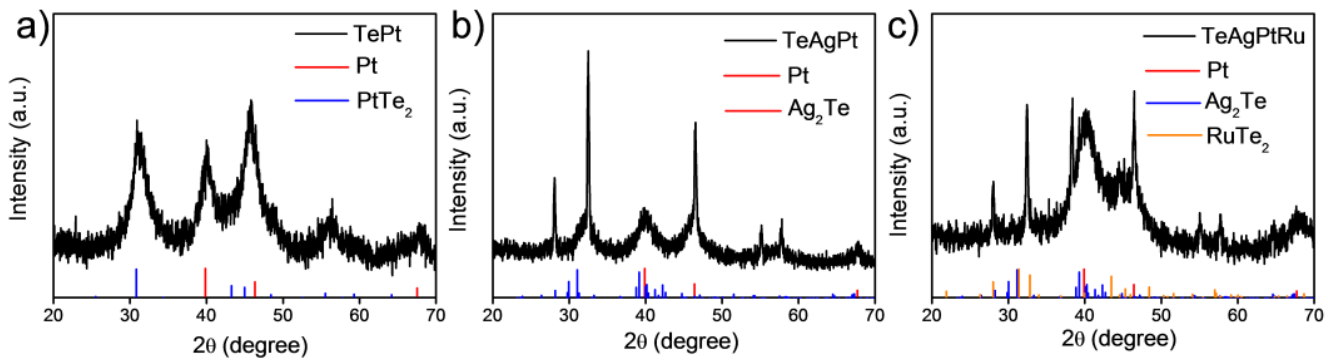

Figure S3. XRD patterns of three 1D Pt-based catalysts. (a) PtTe NWs, (b) PtAgTe NWs and (c) PtRuAgTe NTs.
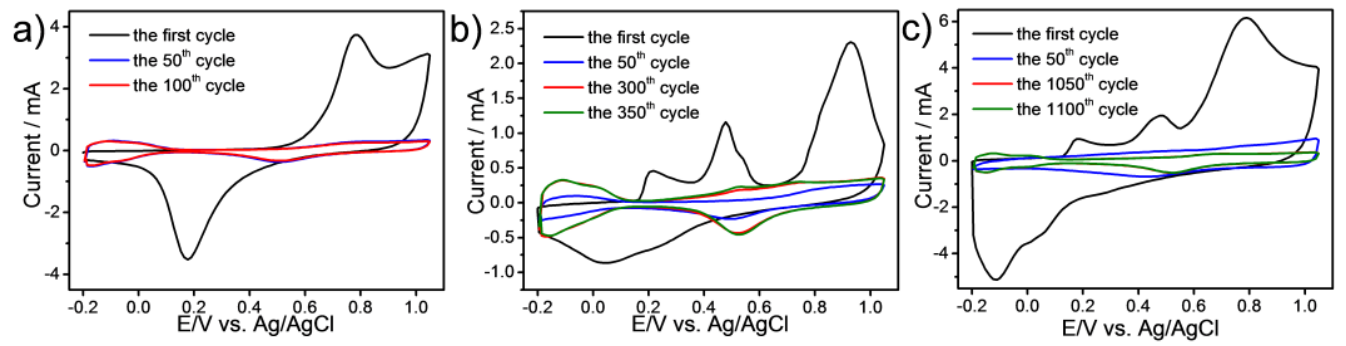

Figure S4. CV profiles of three 1D Pt-based catalysts. (a) PtTe NWs, (b) PtAgTe NWs and (c) PtRuAgTe NTs. CV profiles were recorded in $0.5 \mathrm{M} \mathrm{HClO}_{4}$ solution bubbled with Ar for $30 \mathrm{~min}$ at a sweep rate of $250 \mathrm{mVs}^{-1}$ in the potential of $-0.2-1.05 \mathrm{~V}$. As the figures above displays, PtRuAgTe NTs take the longest time to stabilize in CV scan process, followed by PtAgTe NWs and PtTe NWs, which means more primitive activite sites are available in PtTe 
NWs compared with the other two Pt-based NWs.

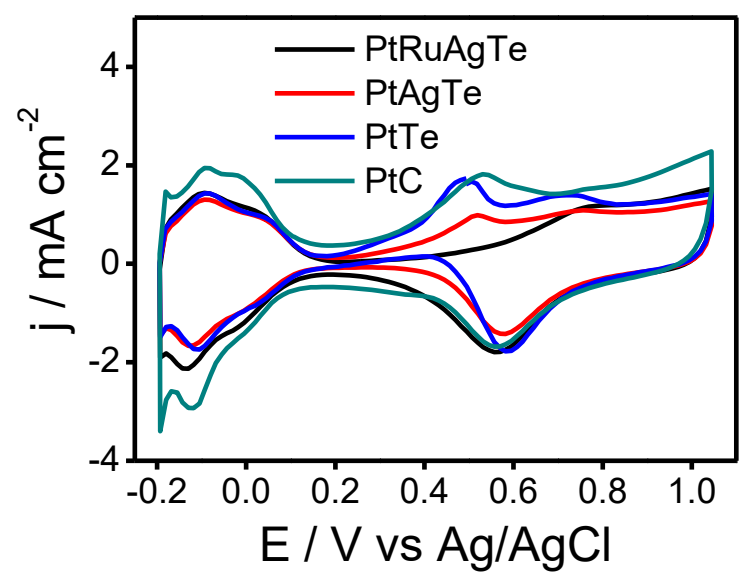

Figure S5. CV profiles of these catalysts for MOR in Ar-saturated $0.5 \mathrm{M} \mathrm{HClO}_{4}$ solution at a sweep rate of $50 \mathrm{mV} \mathrm{s}^{-1}$.

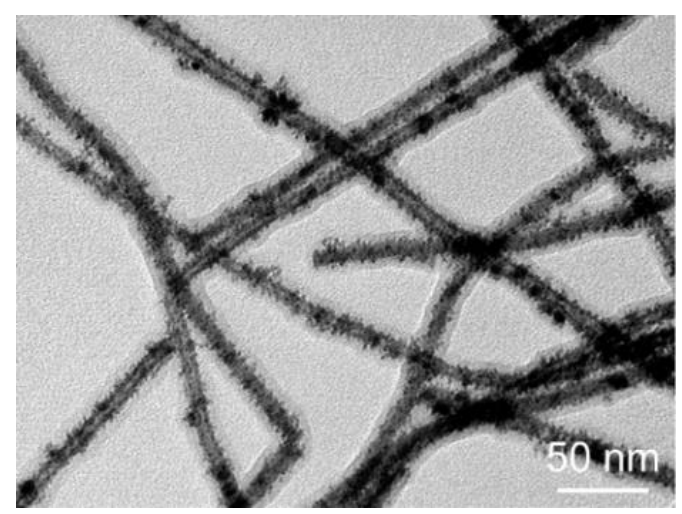

Figure S6. TEM image of PtRuAgTe NTs after CV activation.
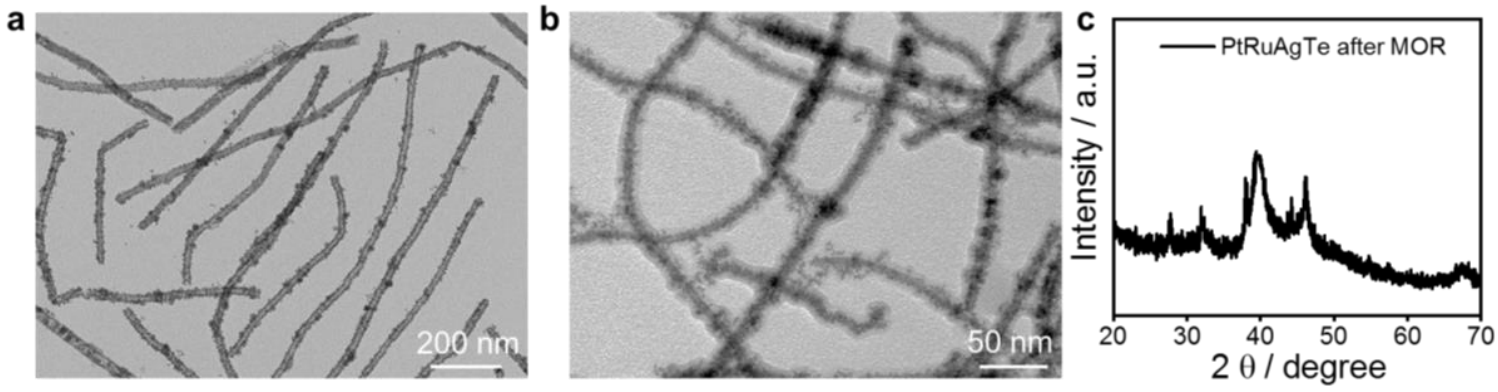

Figure S7. TEM images of PtRuAgTe NTs (a) after MOR measurement and (b) a stability cycle of chronoamperometry. (c) XRD pattern of PtRuAgTe NTs after MOR measurement. 


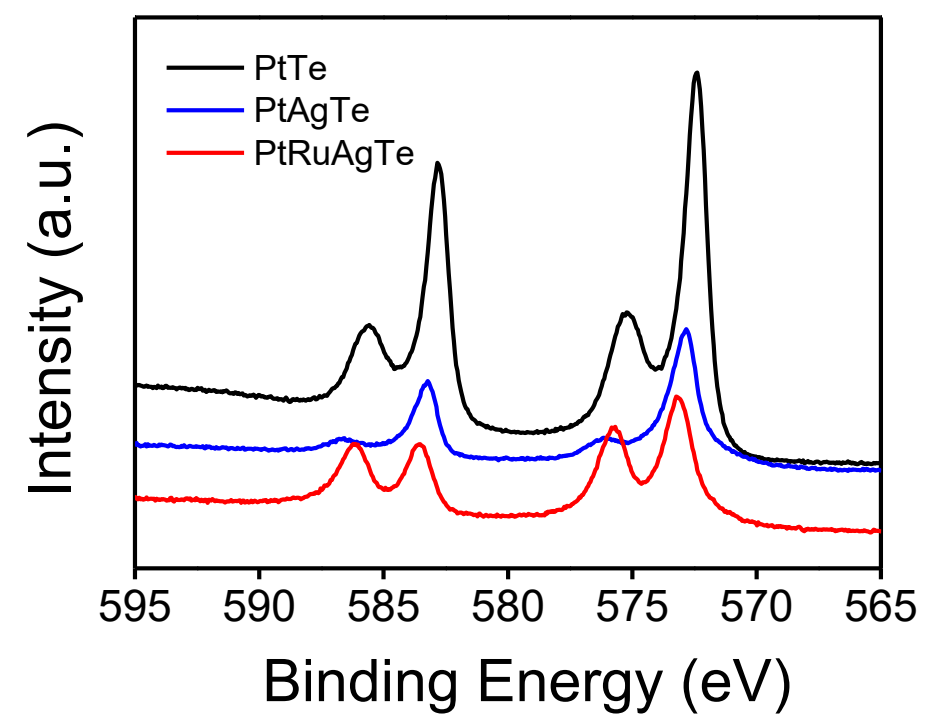

Figure S8. XPS of Te 3d orbital in three 1D Pt-based catalysts. It can be seen from the figure above that element Te existed in two forms, $\mathrm{Te}^{0}$ and $\mathrm{Te}^{4+}$, in PtTe and PtRuAgTe NTs, and adopted dominately the form of $\mathrm{Te}^{0}$ in PtAgTe NWs.
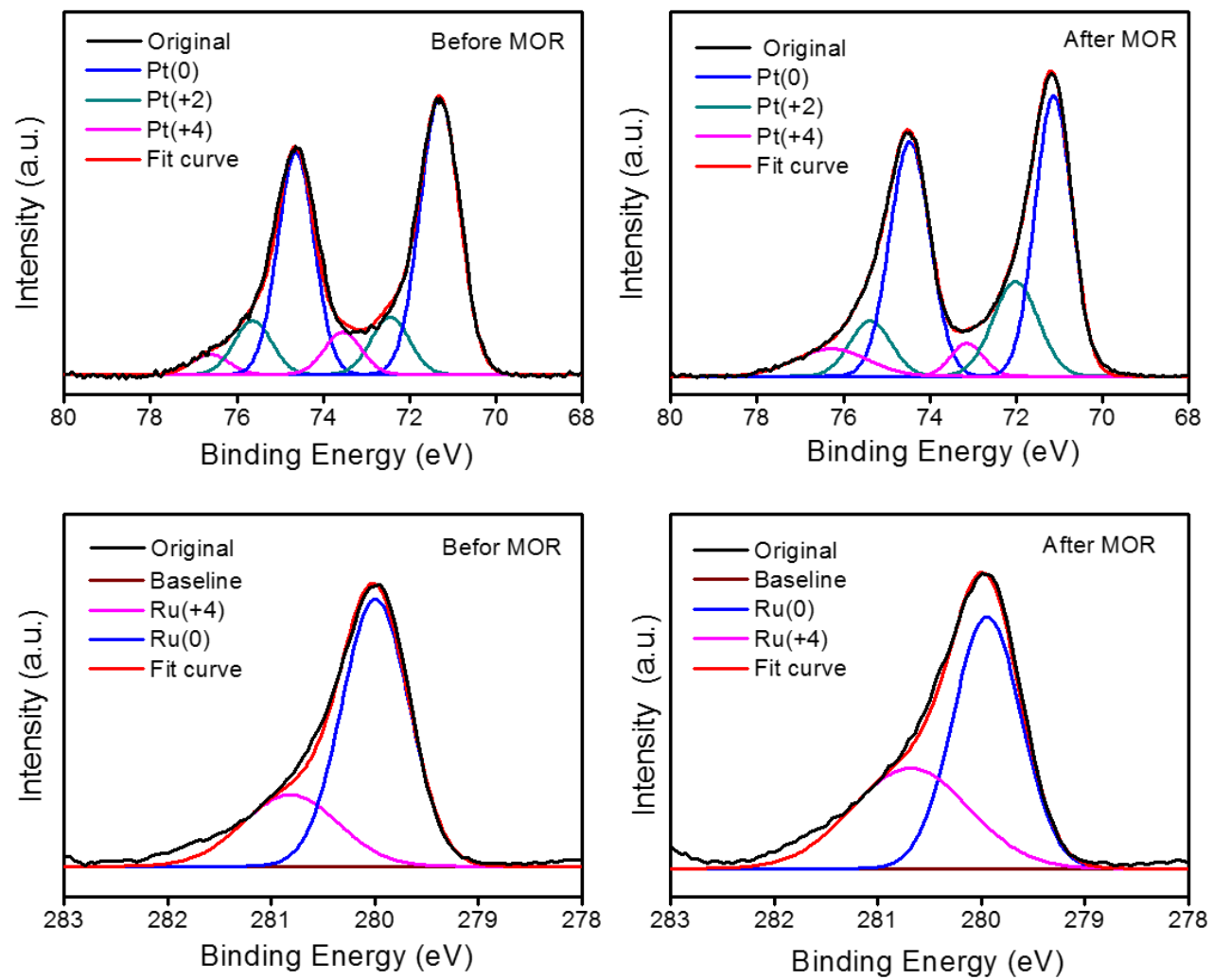

Figure S9. High resolution synchrotron radiation photoelectron spectroscopy. (a, b) $\mathrm{Pt} 4 \mathrm{f}$ and (c, d) Ru 2p orbital of PtRuAgTe NTs before and after MOR. 
Table S1. Electrochemical performance for MOR.

\begin{tabular}{|c|c|c|c|}
\hline Catalyst type & $\begin{array}{l}\text { Mass activity } \\
\qquad(\mathbf{m A} / \mathbf{m g})\end{array}$ & $\begin{array}{l}\text { Specific } \\
\text { activity } \\
\left(\mathbf{m A} / \mathbf{c m}^{2}\right)\end{array}$ & Reference \\
\hline $\mathrm{PtCu}$ NTs & 2252 & 6.09 & $\begin{array}{c}\text { Energy Environ. Sci. 2017, 10, } \\
1751\end{array}$ \\
\hline PtCo NWs & 1020 & 1.95 & Nat. Commun. 2016, 7,11850 \\
\hline PtPdRuTe NTs & 1262 & 2.96 & $\begin{array}{l}\text { J. Am.Chem. Soc. 2017, 139, } \\
5890\end{array}$ \\
\hline Ultrathin Pt NWs & $\sim 500$ & $\sim 1.1$ & $\begin{array}{c}\text { J. Am.Chem. Soc. 2013, 135, } \\
9480\end{array}$ \\
\hline PtPd hollow NPs & 580 & 1.36 & $\begin{array}{c}\text { J. Am.Chem. Soc. 2013, 135, } \\
16762\end{array}$ \\
\hline $\mathrm{PtZn/MWNT}$ & $\sim 580$ & $\sim 1$ & $\begin{array}{c}\text { J. Am.Chem. Soc. 2017, 139, } \\
4762\end{array}$ \\
\hline $\begin{array}{l}\mathrm{PtPd} / \mathrm{Pt} \\
\text { nanoplates }\end{array}$ & 1500 & $\sim 2.6$ & Science, 2016, 354, 1410 \\
\hline PtRu/TiWC NPs & -- & $\sim 1.8$ & Science, 2016, 352, 974 \\
\hline $\mathrm{PtCu}$ icosahedra & 736 & 2.14 & Acs Nano, 2015, 9, 7634 \\
\hline $\mathrm{PtCu}$ wavy NWs & 635 & 2.8 & Chem. Sci. 2016, 7, 5414 \\
\hline $\begin{array}{l}\text { Core/shell } \\
\mathrm{Au} / \mathrm{CuPt} \text { NPs }\end{array}$ & 411 & 0.76 & $\begin{array}{c}\text { J. Am.Chem. Soc. 2014, 136, } \\
5745\end{array}$ \\
\hline $\begin{array}{l}\mathrm{Pt} @ \mathrm{PtNi} \\
\text { nanoplates }\end{array}$ & 782 & 0.96 & Nano Lett. 2016, 16, 7999 \\
\hline PtRuAgTe NTs & 1145 & 1.82 & This work \\
\hline
\end{tabular}

Table S2. ICP-AES results of three catalysts.

\begin{tabular}{cccccc}
\hline Catalysts & $\mathrm{Te}(\%)$ & $\mathrm{Ag}(\%)$ & $\mathrm{Pt}(\%)$ & $\mathrm{Ru}(\%)$ & Molecular formula \\
\hline PtTe & 40.86 & - & 59.14 & - & $\mathrm{Pt}_{59.14} \mathrm{Te}_{40.86}$ \\
PtAgTe & 32.4 & 23.35 & 44.28 & - & $\mathrm{Pt}_{44.28} \mathrm{Ag}_{23.35} \mathrm{Te}_{32.4}$ \\
PtRuAgTe & 21.41 & 12.56 & 25.92 & 40.11 & $\mathrm{Pt}_{25.92} \mathrm{Ru}_{40.11} \mathrm{Ag}_{12.56} \mathrm{Te}_{21.41}$ \\
\hline
\end{tabular}

